\title{
Full causal dissipative cosmologies with stiff matter
}

\author{
M. K. Mak* and T. Harkd \\ Department of Physics, The University of Hong Kong, Pokfulam Road, Hong Kong
}

(Dated: November 14, 2003)

\begin{abstract}
The general solution of the gravitational field equations for a full causal bulk viscous stiff cosmological fluid, with bulk viscosity coefficient proportional to the energy density to the power $1 / 4$, is obtained in the flat Friedmann-Robertson-Walker geometry. The solution describes a non-inflationary Universe, which starts its evolution from a singular state. The time variation of the scale factor, deceleration parameter, viscous pressure, viscous pressure-thermodynamic pressure ratio, comoving entropy and Ricci and Kretschmann invariants is considered in detail.
\end{abstract}

PACS numbers: 98.80.-k, 04.20.Jb

\section{INTRODUCTION}

Dissipative bulk viscous type thermodynamical processes are supposed to play a crucial role in the dynamics and evolution of the early Universe. There are many processes capable of producing bulk viscous stresses in the early cosmological fluid, like interaction between matter and radiation, quark and gluon plasma viscosity, different components of dark matter, particle creation, strings and topological defects etc. Traditionally, for the description of these phenomena the theories of Eckart [1] and Landau and Lifshitz [2] were used. But Hiscock and Lindblom [3] have shown that the Eckart-type theories suffer from serious drawbacks concerning causality and stability. Regardless of the choice of equation of state, all equilibrium states in these theories are unstable and in addition signals may be propagated through the fluid at velocities exceeding the speed of light. These problems arise due to the first order nature of the theory, i.e., it considers only first-order deviations from the equilibrium. The neglected second-order terms are necessary to prevent non-causal and unstable behavior.

A relativistic consistent second-order theory was found by Israel [4] and developed into what is called transient

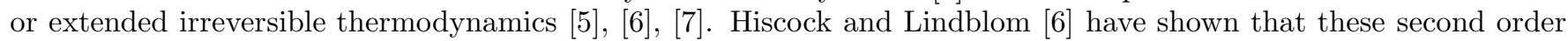
theories are free of the pathologies of the Eckart-type theories. Therefore, the best currently available theory for analyzing dissipative processes in the Universe is the full Israel-Stewart-Hiscock causal thermodynamics.

Exact general solutions of the field equations for flat homogeneous Universes filled with a full causal viscous fluid source for a power-law dependence of the bulk viscosity coefficient on the energy density have been obtained in [8][10]. The evolution of a homogeneous and isotropic dissipative fluid in the truncated Israel-Stewart theory has been analyzed, by using dynamical systems methods, by Di Prisco, Herrera and Ibanez [11]. They have found that almost all solutions inflate, and only a few of them can be considered physical, since they do not satisfy the dominant energy condition.

It is the purpose of the present paper to obtain the general solution of the gravitational field equations for the case of a bulk viscous cosmological fluid in a flat Friedmann-Robertson-Walker type geometry, obeying the Zeldovich (stiff) equation of state, with the bulk viscosity coefficient $\xi$ proportional to the energy density $\rho$ to the power $1 / 4$, $\xi \propto \rho^{1 / 4}$. In this case the solution of the gravitational field equations can be represented in an exact parametric form, in terms of Bessel functions. The behavior of the scale factor, deceleration parameter, viscous pressure, viscous pressure-thermodynamic pressure ratio, comoving entropy and Ricci and Kretschmann invariants is also considered. The solution describes a non-inflationary Universe, which starts its evolution from a singular state.

The present paper is organized as follows. The basic equations describing the dynamics of the viscous FriedmannRobertson-Walker (FRW) Universe are written down in Section II. The general solution of the field equations is obtained in Section III. In Section IV we discuss and conclude our results.

\footnotetext{
*Electronic address: mkmak@vtc.edu.hk

†Electronic address: harko@hkucc.hku.hk
} 


\section{GEOMETRY, FIELD EQUATIONS AND CONSEQUENCES}

The energy-momentum tensor for a bulk viscous cosmological fluid is given by

$$
T_{i}^{k}=(\rho+p+\Pi) u_{i} u^{k}-(p+\Pi) \delta_{i}^{k},
$$

where $\rho$ is the energy density, $p$ the thermodynamic pressure, $\Pi$ the bulk viscous pressure and $u_{i}$ the four velocity satisfying the condition $u_{i} u^{i}=1$. We use units so that $8 \pi G=c=1$.

For a flat Friedmann-Robertson-Walker Universe with a line element given by $d s^{2}=d t^{2}-a^{2}(t)\left(d x^{2}+d y^{2}+d z^{2}\right)$, filled with a bulk viscous cosmological fluid, the gravitational field equations and the continuity equation $T_{i ; k}^{k}=0$ (with ; denoting the covariant derivative with respect to the metric) imply

$$
3 H^{2}=\rho, 2 \dot{H}+3 H^{2}=-p-\Pi, \dot{\rho}+3(\rho+p) H=-3 H \Pi,
$$

where $H=\dot{a} / a$ is the Hubble parameter. The causal evolution equation for the bulk viscous pressure is given by [12]

$$
\tau \dot{\Pi}+\Pi=-3 \xi H-\frac{1}{2} \tau \Pi\left(3 H+\frac{\dot{\tau}}{\tau}-\frac{\dot{\xi}}{\xi}-\frac{\dot{T}}{T}\right),
$$

where $T$ is the temperature, $\xi$ the bulk viscosity coefficient and $\tau$ the relaxation time. Eq. (3) arises as the simplest way (linear in $\Pi$ ) to satisfy the $H$ theorem ( i.e., for the entropy production to be non-negative, $S_{; i}^{i}=\Pi^{2} / \xi T \geq 0$, where $S^{i}=e N^{i}-\frac{\tau \Pi^{2}}{2 \xi T} u^{i}$ is the entropy flow vector, $e$ is the entropy per particle and $N^{i}=n u^{i}$ is the particle flow vector) [5], [6].

In order to close the system of equations (2) we have to give the equation of state for $p$ and specify $T, \xi$ and $\tau$. As usual, we assume the following phenomenological laws [12]:

$$
p=(\gamma-1) \rho, \xi=\alpha \rho^{s}, T=\beta \rho^{r}, \tau=\xi \rho^{-1}=\alpha \rho^{s-1},
$$

where $1 \leq \gamma \leq 2$, and $\alpha \geq 0, \beta \geq 0, r \geq 0$ and $s \geq 0$ are constants. Eqs. (4) are standard in cosmological models whereas the equation for $\tau$ is a simple procedure to ensure that the speed of viscous pulses does not exceed the speed of light.

The requirement that the entropy is a state function imposes in the present model the constraint $r=(\gamma-1) / \gamma[\underline{8}]$ so that $0 \leq r \leq 1 / 2$ for $1 \leq \gamma \leq 2$.

The growth of the total comoving entropy $\Sigma$ over a proper time interval $\left(t_{0}, t\right)$ is given by [12]:

$$
\Sigma(t)-\Sigma\left(t_{0}\right)=-3 k_{B}^{-1} \int_{t_{0}}^{t} \Pi H a^{3} T^{-1} d t,
$$

where $k_{B}$ is the Boltzmann constant. The Israel-Stewart-Hiscock theory is derived under the assumption that the thermodynamical state of the fluid is close to equilibrium, that is the non-equilibrium bulk viscous pressure should be small when compared to the local equilibrium pressure $|\Pi|<<p=(\gamma-1) \rho$ [13]. If this condition is violated then one is effectively assuming that the linear theory holds also in the nonlinear regime far from equilibrium. For a fluid description of the matter, the condition ought to be satisfied.

To see if a cosmological model inflates or not it is convenient to introduce the deceleration parameter $q=$ $d\left(H^{-1}\right) / d t-1=(\rho+3 p+3 \Pi) / 2 \rho$. The positive sign of the deceleration parameter corresponds to standard decelerating models, whereas the negative sign indicates inflation.

With these assumptions the evolution equation for the flat homogeneous causal bulk viscous cosmological models is

$$
\ddot{H}+3 H \dot{H}+3^{1-s} \alpha^{-1} H^{2-2 s} \dot{H}-(1+r) H^{-1} \dot{H}^{2}+\frac{9}{4}(\gamma-2) H^{3}+\frac{3^{2-s}}{2 \alpha} \gamma H^{4-2 s}=0 .
$$

By introducing a set of non-dimensional variables $h$ and $\theta$ by means of the transformations $H=\alpha_{0} h, t=\frac{2}{3 \alpha_{0}} \theta$, with $\alpha_{0}=\left(\frac{3^{s} \alpha}{2}\right)^{\frac{1}{1-2 s}}, s \neq \frac{1}{2}$, and using the expression of $r$ as a function of $\gamma$, Eq. (6) takes the form

$$
\frac{d^{2} h}{d \theta^{2}}+\left[2 h+h^{2(1-s)}\right] \frac{d h}{d \theta}-(1+r) h^{-1}\left(\frac{d h}{d \theta}\right)^{2}+\frac{2 r-1}{1-r} h^{3}+\frac{1}{1-r} h^{2(2-s)}=0 .
$$

By denoting $n=(1-2 s) /(1-r)$ and changing the variables according to $h=y^{1 /(1-r)}, \eta=\int y^{1 /(1-r)} d \theta$, Eq. (7) becomes:

$$
\frac{d^{2} y}{d \eta^{2}}+\left(2+y^{n}\right) \frac{d y}{d \eta}+(2 r-1) y+y^{n+1}=0
$$




\section{GENERAL SOLUTION FOR A STIFF COSMOLOGICAL FLUID WITH $s=1 / 4$}

The stiff cosmological fluid, with equilibrium pressure equal to the energy density, is supposed to give an accurate description of the very early phases of the evolution of the Universe. It corresponds to the values of the parameters $\gamma=2$ and, consequently, $r=1 / 2$. We assume that the bulk viscosity coefficient $\xi$ is of the form $\xi=\alpha \rho^{1 / 4}$, with $s=1 / 4$. Hence it follows that $n=1$. Therefore the nonlinear second order ordinary differential equation (8) becomes:

$$
\frac{d^{2} y}{d \eta^{2}}+(2+y) \frac{d y}{d \eta}+y^{2}=0
$$

A particular solution of Eq. (9) for $n=1, s=1 / 4$ has been obtained in [14]. The general solution of Eq. (9) is given by

$$
y(\eta)=\frac{2 C_{1} e^{-\eta}\left[J_{1}\left(C_{1} e^{-\eta}\right)+C_{2} Y_{1}\left(C_{1} e^{-\eta}\right)\right]}{C_{2} Y_{0}\left(C_{1} e^{-\eta}\right)+J_{0}\left(C_{1} e^{-\eta}\right)},
$$

where $C_{1}$ and $C_{2}$ are arbitrary integration constants, $J_{k}(x)$ and $Y_{k}(x)$ are the Bessel functions of the first and second kinds, respectively. They satisfy the Bessel differential equation

$$
x^{2} \frac{d^{2} f}{d x^{2}}+x \frac{d f}{d x}+\left(x^{2}-k^{2}\right) f=0 .
$$

Hence the general solution of the gravitational field equations for a Zeldovich causal bulk viscous fluid filled flat FRW Universe, with bulk viscosity coefficient $\xi=\alpha \rho^{1 / 4}$, can be represented in the following exact parametric form, with $\eta$ taken as parameter:

$$
\begin{gathered}
t(\eta)-t_{0}=\frac{2}{3 \alpha_{0}} \int \frac{d \eta}{y^{2}(\eta)}, H(\eta)=\alpha_{0} y^{2}(\eta), a(\eta)=a_{0} \exp \left(\frac{2}{3} \eta\right), \rho(\eta)=p(\eta)=3 \alpha_{0}^{2} y^{4}(\eta) \\
q(\eta)=-\frac{3}{y(\eta)} \frac{d y}{d \eta}-1, \Pi(\eta)=-6 \alpha_{0}^{2} y^{3}(\eta)\left[y(\eta)+\frac{d y}{d \eta}\right],\left|\frac{\Pi}{p}\right|=2\left|1+\frac{d \ln y}{d \eta}\right| \\
\Sigma(\eta)=\Sigma\left(\eta_{0}\right)+\frac{4 \sqrt{3} \alpha_{0} a_{0}^{3}}{k_{B} \beta} \int y(\eta)\left[y(\eta)+\frac{d y}{d \eta}\right] \exp (2 \eta) d \eta
\end{gathered}
$$

where $a_{0}, t_{0}$ and $\Sigma\left(\eta_{0}\right)$ are constants of integration and $\alpha_{0}=2 / 3 \alpha$.

The singular or non-singular character of the solution for all times $t \geq 0$ can be checked from the finite (infinite) character of the Ricci invariant $R_{i j} R^{i j}$ and Kretschmann scalar $R_{i j k l} R^{i j k l}$, given by

$$
\begin{gathered}
I=R_{i j} R^{i j}=12\left[\left(\frac{\ddot{a}}{a}\right)^{2}+\frac{\ddot{a}}{a}\left(\frac{\dot{a}}{a}\right)^{2}+\left(\frac{\dot{a}}{a}\right)^{4}\right]=36 \alpha_{0}^{4} y^{6}\left[3\left(\frac{d y}{d \eta}\right)^{2}+3 y \frac{d y}{d \eta}+y^{2}\right], \\
J=R_{i j k l} R^{i j k l}=12\left[\left(\frac{\ddot{a}}{a}\right)^{2}+\left(\frac{\dot{a}}{a}\right)^{4}\right]=12 \alpha_{0}^{4} y^{6}\left[9\left(\frac{d y}{d \eta}\right)^{2}+6 y \frac{d y}{d \eta}+2 y^{2}\right] .
\end{gathered}
$$

\section{DISCUSSIONS AND FINAL REMARKS}

The evolution of the causal bulk viscous Zeldovich fluid filled flat Universe starts generally its evolution from a singular state, as can be seen from the singular behavior of the invariant $R_{i j} R^{i j}$, represented, for different values of the integration constants $C_{1}$ and $C_{2}$, in Fig.1.

Generally, the dynamics of the Universe depends on the numerical values of the constants $C_{1}$ and $C_{2}$. The behavior of the scale factor is presented in Fig.2, for some specific values of the integration constants. The evolution is expansionary for all times. 


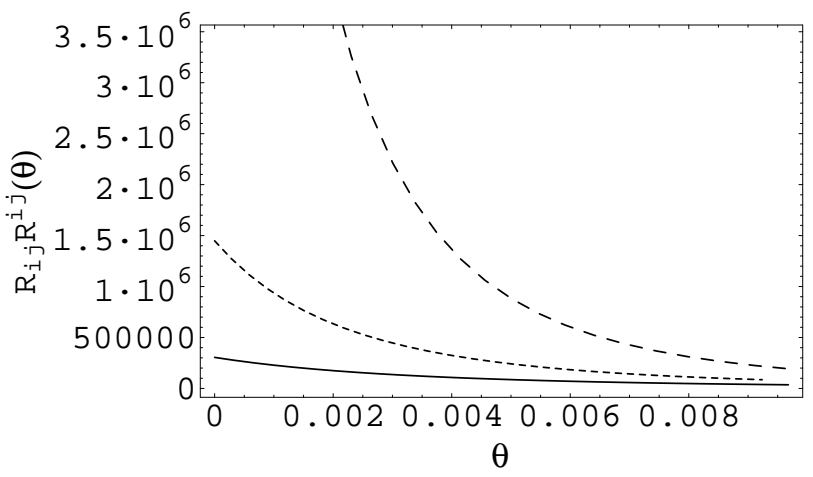

FIG. 1: Time evolution of the invariant $R_{i j} R^{i j}$ for different values of the integration constants $C_{1}$ and $C_{2}: C_{1}=1$ and $C_{2}=-1$ (solid curve), $C_{1}=1.1$ and $C_{2}=-0.9$ (dotted curve) and $C_{1}=1.1$ and $C_{2}=-1$ (dashed curve).

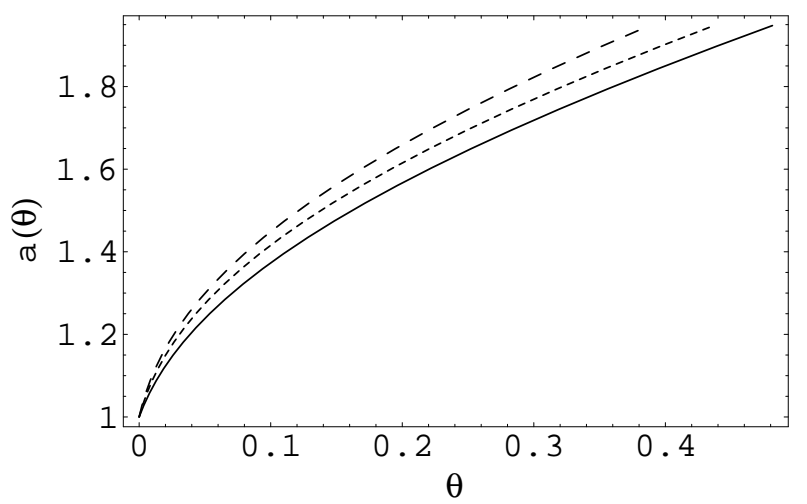

FIG. 2: Time variation of the scale factor $a$ for different values of the integration constants $C_{1}$ and $C_{2}: C_{1}=1$ and $C_{2}=-1$ (solid curve), $C_{1}=1.1$ and $C_{2}=-0.9$ (dotted curve) and $C_{1}=1.1$ and $C_{2}=-1$ (dashed curve).

The variation of the energy density of the cosmological fluid is represented in Fig. 3.

At the initial moment the scale factor is zero, while the energy density tends to infinity.

The dynamics of the deceleration parameter, shown in Fig.4, indicates, for the chosen range of the integration constant, a non-inflationary behavior for all times, with $q>0, \forall t \geq 0$.

The bulk viscous pressure $\Pi$ is negative during the cosmological evolution, $\Pi<0, \forall t \geq 0$, as expected from a thermodynamic point of view. In the large time limit the viscous pressure tends to zero. In the same limit the bulk viscosity coefficient also becomes negligible small. During the cosmological evolution a large amount of comoving entropy is produced, with the entropy $\Sigma$ increasing in time and tending in the large time limit to a constant value. The time variation of the entropy is presented in Fig. 5.

The ratio of the bulk and thermodynamic pressures, $|\Pi / p|$ is presented in Fig. 6 .

For the chosen values of the integration constants the condition $\Pi / p$ does not hold for all times, and therefore the model is thermodynamically consistent only for a finite period of time. Therefore the solution represented by Eqs. (12)-(14) can consistently describe only a well-determined, non-inflationary period of the early dynamics of the super-dense, matter dominated post-inflationary era, when, as expected, the bulk viscous dissipative effects play an important role.

[1] C. Eckart, Phys. Rev. 58, 919 (1940). 


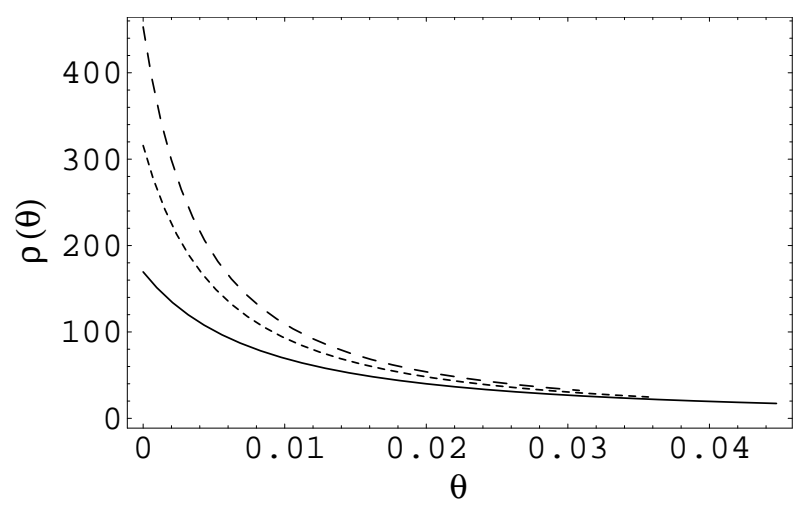

FIG. 3: Evolution of the energy density $\rho$ for different values of the integration constants $C_{1}$ and $C_{2}$ : $C_{1}=1$ and $C_{2}=-1$ (solid curve), $C_{1}=1.2$ and $C_{2}=-0.8$ (dotted curve) and $C_{1}=1.1$ and $C_{2}=-1.8$ (dashed curve).

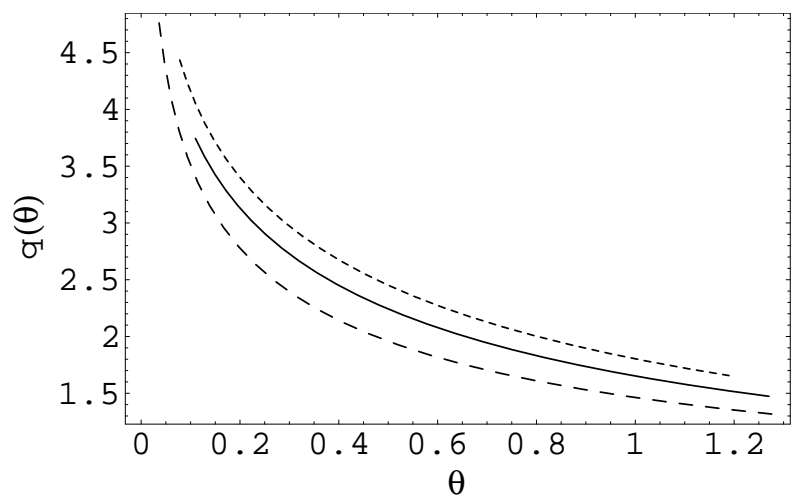

FIG. 4: Dynamics of the deceleration parameter $q$ for different values of the integration constants $C_{1}$ and $C_{2}$ : $C_{1}=1$ and $C_{2}=-1$ (solid curve), $C_{1}=1.2$ and $C_{2}=-0.8$ (dotted curve) and $C_{1}=1.1$ and $C_{2}=-1.8$ (dashed curve).

[2] L. D. Landau, E. M. Lifshitz, Fluid Mechanics, (Butterworth Heinemann, Oxford, 1987).

[3] W. A. Hiscock, L. A. Lindblom, Phys. Rev. D31, 725 (1985).

[4] W. Israel W., Ann. Phys. 100, 310 (1976).

[5] W. Israel, J. M. Stewart, Phys. Lett. A58, 213 (1976).

[6] W. A. Hiscock, L. Lindblom, Ann. Phys. 151, 466 (1989).

[7] W. A. Hiscock, J. Salmonson, Phys. Rev. D43, 3249 (1991).

[8] L. P. Chimento, A. S. Jakubi, Class. Quantum Grav. 14, 1811 (1997).

[9] L. P. Chimento, A. S. Jakubi, Int. J. Mod. Phys. D7, 177 (1998) .

[10] M. K. Mak, T. Harko, Gen. Rel. Grav. 30, 1171 (1998); M. K. Mak, T. Harko, Gen. Rel. Grav. 31, 273 (1999); M. K. Mak, T. Harko, J. Math. Phys. 39, 5458 (1998) ; M. K. Mak, T. Harko, Int. J. Theor. Phys. 38, 1561 (1999) ; M. K. Mak, T. Harko, Aust. J. Phys. 52, 659 (1999); M. K. Mak, T. Harko, Int. J. Mod. Phys. D9, 97 (2000); M. K. Mak, T. Harko, Int. J. Mod. Phys. D9, 475 (2000) ; M. K. Mak, T. Harko, Europhys. Lett. 56, 762 (2001); C.-M. Chen, T. Harko, M. K. Mak, Phys. Rev. D64, 124017 (2001); T. Harko, M. K. Mak, Class. Quantum Grav. 20, 407 (2003); I. Brevik, Int. J. Mod. Phys. A18, 2145 (2003); I. Brevik and A. Hallanger, gr-qc/0308058

[11] A. Di Prisco, L. Herrera, J. Ibanez, Phys. Rev. D63, 023501 (2001).

[12] R. Maartens, Class. Quantum Grav. 12, 1455 (1995).

[13] W. Zimdahl, Phys. Rev. D53, 5483 (1996); R. Maartens, V. Mendez, Phys. Rev. D55, 1937 (1997).

[14] M. K. Mak, T. Harko, Aust. J. Phys. 53, 241 (2000). 


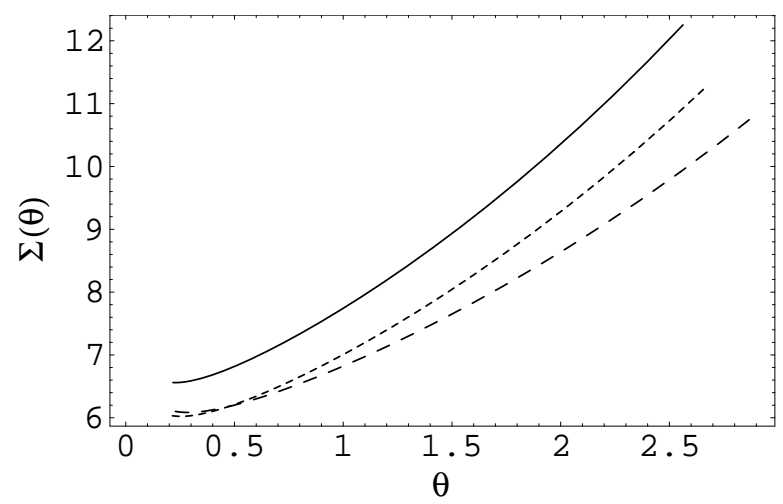

FIG. 5: Behavior of the comoving entropy $\Sigma$ for different values of the integration constants $C_{1}$ and $C_{2}$ : $C_{1}=1$ and $C_{2}=-1$ (solid curve), $C_{1}=1.05$ and $C_{2}=-0.9$ (dotted curve) and $C_{1}=1.08$ and $C_{2}=-0.8$ (dashed curve). The numerical parameters have been normalized so that $4 \sqrt{3} \alpha_{0} a_{0}^{3} / k_{B} \beta=1$.

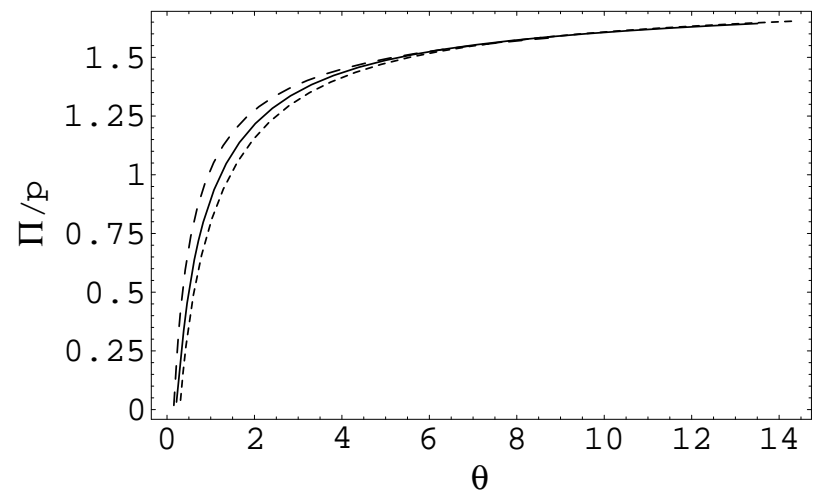

FIG. 6: Variation with time of the ratio $\frac{\Pi}{p}$ for different values of the integration constants $C_{1}$ and $C_{2}: C_{1}=1$ and $C_{2}=-1$ (solid curve), $C_{1}=1.1$ and $C_{2}=-0.9$ (dotted curve) and $C_{1}=1.2$ and $C_{2}=-0.9$ (dashed curve). 